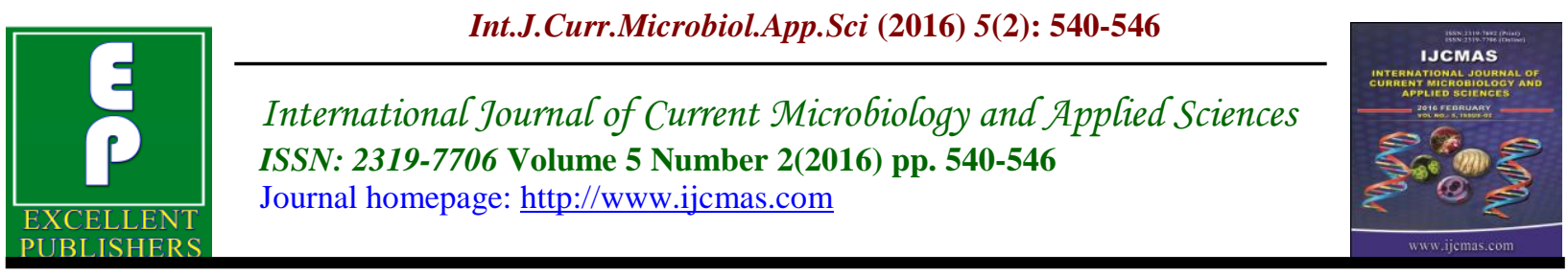

Review Article

doi: http://dx.doi.org/10.20546/ijcmas.2016.502.061

\title{
Understanding of Quorum - Sensing: A Possible Solution for Drug Resistance in Bacteria
}

\author{
Seema Bose ${ }^{1 *}$ and Atindra Krishna Ghosh ${ }^{2}$ \\ Department of Microbiology, Rural medical college, Pravara Institute of Medical Sciences, \\ Loni, Maharashtra, India \\ Department of Medicine, Rural Medical College, Pravara Institute of Medical Sciences, \\ Loni, Maharashtra, India \\ *Corresponding author
}

\begin{tabular}{|c|c|}
\hline & A B S T R A C T \\
\hline & \multirow{4}{*}{$\begin{array}{l}\text { Quorum - sensing (QS) enables bacteria to establish cell- to - cell } \\
\text { communication. Few among many QS molecules in bacteria are peptide and } \\
\text { acylated homoserine lactones. QS regulate genes that code for invasion, } \\
\text { spread and other virulence factors. It also takes part in biofilm formation. } \\
\text { QS mediate via small signaling molecules known as auto inducers. Quorum } \\
\text { - quenching inhibits QS. Several chemical compounds and enzymes mediate } \\
\text { inhibition of QS, such as, lactonases, acylases and oxydoreductases. Other } \\
\text { than these, there are some non-enzymatic methods for quorum quenching. } \\
\text { Blocking of QS by QS inhibition (QSI) may play an important role to } \\
\text { disrupt biofilm formation in a device - associated infection and chronic drug } \\
\text { resistant infection. More researches are required in this area related with QS } \\
\text { and QSI. }\end{array}$} \\
\hline $\begin{array}{l}\text { Quorum - } \\
\text { sensing } \\
\text { Quorum - } \\
\text { quenching }\end{array}$ & \\
\hline Article & \\
\hline $\begin{array}{l}\text { Accepted: } \\
\text { 20 January } 2016 \\
\text { Available Online: } \\
\text { 10, February } 2016\end{array}$ & \\
\hline
\end{tabular}

\section{Introduction}

Quorum - sensing (QS) is a cell-to-cell communication in bacteria, which leads to gene expression within a bacterial community. Using QS, bacteria regulate those genes involved in invasion and spread of the pathogens. It can occur in a group of a same bacterial species as well as, between different species. The possible role of understanding of QS and its inhibition in the treatment of infective disorders is promising.
The present day therapy against infectious disease is mostly dependent on antibiotic treatment. Antibiotic therapy elicits strong selective pressure on bacteria and thereby, development of drug resistance mechanism in them. Blocking cell-to-cell communication by QS may act as an important tool to disturb their group action. QS depend on secretion of small signaling molecules by bacteria. Sometimes it directly 
causes synchronized production of proteases, toxins and other substances for their own defense mechanism. It may cause biofilm formation and bacterial drug resistance. Bacteria can act more powerfully in a community than the individual bacterial cell. Inhibition of QS does not suppress growth of bacterial cell and thereby, will not exert a selective pressure on them to develop antibiotic resistance. QS inhibition (QSI) or Quorum - quenching is a process, which inhibits QS by disrupting signaling and degrading signaling molecule (Miller et al., 2001; Lerat et al., 2004; Chan et al., 2011).

\section{Mechanisms of Quorum -Sensing and Quorum-Quenching}

QS in bacteria were first time observed in a bioluminescent microorganism, Aliivibrio fischeri (Nealson et al., 1979). In QS system secretions of small pheromone like signaling molecules play a major role. These molecules are known as auto inducers (AIs). First time in the year 2001, three dimensional structure of protein molecule responsible for QS was reported (Lewis et al., 2001). Broadly, auto inducers can be classified into following types: (LaSarre et al., 2013).

Acylated homoserine lactones (AHLs or AI 1) - mostly found in Gram - negative bacteria (GNB).

Peptides - used by Gram- positive bacteria (GPB). Quorumpep is a database of QS peptides.

Auto inducer 2- used by both GNB and GPB.

Others - few among many examples of these are Pseudomonas quinolone signal (PQS), diffusible signal factor (DSF) and auto inducer - 3 (Wynendaele et al., 2012).
In QS system, microorganisms produce AIs and pheromones. Bacterial receptors bind with AIs. This binding activates transcription of some specific genes. These genes code for inducer synthesis also. A bacterial cell must receive signal by inducers by some other cells (not by its own). This causes activation of certain gene transcription. The concentration of inducer is directly proportional to the growth or density of bacterial population. One of the common examples is bioluminescent luciferase gene produced by Vibrio fischeri. Transcription of luciferase is induced leading to bioluminescence. It has been observed that when luciferase gene presented by a single cell, bioluminescence will not be visible. For this observable phenomenon, coordinated behavior of bacterial cells in a community is required. On the other hand, very large number of bacterial cells can limit the production of luciferase and by doing so; can avoid expenditure of energy on the production of useless product (David et al., 2005). Serratia liquifaciens (S. liquifaciens) MGI strain possesses $\mathrm{N}$ - acylhomoserine lactone which regulate QS system. This regulated QS system in turn, controls colonization of organism by swarming. There are two QS regulated genes found in S. liquifaciens MGI strain, such as, $b s m A$ and $b s m B$. These genes encode for biofilm formation. Bacterial QS regulate bioluminescence, competence, antibiotic production and secretion of virulence factor. Firstly, there is production of signaling molecules, such as, AIs by bacterial community. These AIs are detected by receptors present in cytoplasm or on cell membrane. Detection of AIs causes activation of genes responsible for behavior of bacteria as a community. These also cause further activation of AIs production (Labbale et al., 2004).

QS systems of GPB and GNB bacteria are 
different. In case of GPB, auto-inducing peptides (AIPs) act as signaling molecules. AIP is produced in the cell, processed further and then secreted from the cells. Extracellular AIPs in high concentration bind with Histidine kinase receptors. This binding activates kinase activity of receptor; auto phosphorylates it and passes phosphate to cytoplasmic response regulator. Finally, phosphorylated response regulator stimulates transcription of genes involved with QS system.

GNB generally use AHLs or other molecules for QS. The production of AHLs depends on $\mathrm{S}-$ adenosylmethionine (SAM). This SAM is used as substrate. GNB produce AIs, which are freely diffused through cell membrane. AIs bind with cytoplasmic receptor at optimum concentration. This binding causes regulation of expression of genes involved in QS (Rutherford et al., 2012).

In some cases, AIs produced by GNB are detected by Histidine kinase receptors. In that cases, gene regulation involved in QS system follow the same mechanism as in GPB involved in QS (Rutherford et al., 2012). The signaling activities of Pseudomonas aeruginosa (P.aeruginosa) are perhaps most studied among all microorganisms. There are several reports on cell- to - cell signaling process of bacteria. The signaling molecules described in these studies are AHL, 4 - quinolones, the global activator of antibiotic and cyanide synthesis (GAC), cyclic AMP (C AMP), cyclic di - GMP (C - di - GMP), guanosine pentaphosphate (ppp Gpp) and alarmones guanosine tetra phosphate (ppGpp). There are very few reports on less studied signaling molecules, such as, pyoverdin, pyocyanin, diketopiperazines and fatty acid like factors (Jimenez et al., 2012).
A two - component response systems (TCRSs) is one of the major requirements for bacterial or fungal virulence. This is defined as the ability to express a set of genes in response to a signal specific for a particular environment. TCRSs are a very important component of QS system in GPB. This depends on bacterial density and is named as accessory gene regulator (agr). The agr system also regulates biofilm formation (Mayville et al., 1999; Otto et al., 2004). Several TCRSs are responsible for stimulating gene expression and microbial resistance to antibiotics (Arthur et al., 1992; Evers et al., 1996).

\section{Inactivation of QS (Quorum - quenching)}

The process inhibits QS by disrupting cellto-cell communication. Disrupting signal and degrading signaling molecules achieve this. There are several molecules, which can cause inactivation of QS. Quorum quenching enzymes was first time identified in Bacillus species (Dong et al., 2000). Three known quorum - quenching enzymes, such as, lactonases, acylases and oxydoreductases can degrade AHL signals (Jimenez et al., 2012; Kim et al., 2005). Other than that, certain AHL - degrading loci were found in Rhizobium species (strain NGR 234). Following genes of this species elicited quorum - quenching ability: $d h l R$, $q s d R 1$, qsdR2 and hydR - hitR (Maria et al., 2007; Bokhove et al., 2010; Lead better et al., 2000; Uroz et al., 2005). There are also non-enzymatic methods of QSI, such as, use of antibodies. Use of monoclonal antibodies (MAbs) against AHLs is one of the many examples. Another example is immunization of bovine serum albumin (BSA) conjugated $30 \mathrm{c} 12 \mathrm{hsl}$ in a mouse intranasally challenged with Pseudomonas aeruginosa. In that case, bacterial load remains the same (Kaufmann et al., 2008; Kaufmann et al., 2006; Miyairi et al., 2006., Parsek et al., 1999). One of less 
studied method for QSI is QS signal biosynthesis. AHL signals are generated by acyl - HSL synthases. These synthases belong to the Lux 1 or Ains family. They are produced from the substrate SAM and acylACP. The AHL production can be inhibited by suppression of biosynthesis of SAM, interference with production of acyl - ACPs and inactivation of synthase (Gilson et al., 1995; Cugini et al., 2007). Several researches are also going on world - wide to inhibit cell - to - cell signaling in bacterial community by production of antibodies. These antibodies bind with signaling molecules and inhibit cell - to - cell communication. This shows promising result in case of GPB. Development of antibodies in case of GPB is favored because of presence of peptides involving in QS. Some workers in their research work used AHLs and AHL analogue conjugated to keyhole limpet hemocyanin (KLH) or bovine serum albumin (BSA). This caused production of AHL sequestering antibodies that protected experimental mice against P.aeruginosa infection (Kaufmann et al., 2008; Kaufmann et al., 2006; Miyairi et al; 2005).

Scope of use of antimicrobial drugs for inhibition of QS is also being explored. One of the examples of these is farnesol. This farnesol can be effective to target 4 quinolone - mediated signaling in P.aeruginosa (Cugini et al; 2007).

Quorum - quenching is not bactericidal. Therefore, development of drug resistance is not found in this therapeutic method (Rasko et al; 2010). Bacterial viability is not affected by blockage of QS. So, combined therapy with QS inhibitors plus antibiotics may be recommended for treatment of persistent infection. Some QS inhibitors can increase sensitivity of certain antibiotics but this property has been rarely tested (Kiran, S.; 2011).
There are also some bacteria that carry more than one QS system. So disruption of one QS system may not prevent expression of other virulence factors communicated by other QS systems (Dekimpe et al; 2009). There are 3 parallel QS systems identified in Vibrio cholerae among which 2 are responsible for colonization on host cell and production of virulence factors, such as, cholera toxin and toxin co - regulated pilus (Miller et al; 2002).

Biofilms can be formed on abiotic medical devices, such as, catheters and surgical implants. Once formed, this biofilm is difficult to remove and cause persistent chronic infectious disease (Saye, D.E.; 2007). several strategies are undertaken to inhibit biofilm formation and removal of already established one. In this regard, QS inhibitors are showing promising result. Inhibition of QS system requires interruption of gene expression involved in biofilm formation (Benghezal et al, 2007; Bose et al, 2011; Bose et al, 2015). Besides, this also requires inhibition of biosynthesis of the polysaccharide and proteinaceous component of biofilm and identification and manipulation of enzymes that can break down the biofilm mass (Song et al, 2013). At present; only few reports are available about QS in Acinetobacter baumannii, Klebsiella pneumoniae and Enterobacter species. There are some reports against quorum - quenching therapy leading to an increase in resistant mutant strain of bacteria (Song et al, 2013). There are some non conventional therapies to overcome persistent infections, such as, use of AMPs, polyphenols, bacteriophages and nano antibiotics. These types of therapies kill microorganisms without inducing evolutionary stress, therefore, decreasing the chances of development of drug resistance (Ivanova et al, 2013). 
In conclusion, Many works had been done to inhibit quorum molecule synthesis, accumulation and signaling against many pathogenic bacteria. GNB are the most significant pathogens causing health care associated infections.

A lot of work had been done to study the QS and quorum quenching mechanisms of $P$. aeruginosa. But only few reports are available for some other bacteria. Further research is necessary to explore this grey zone or less studied area.

Disadvantage of non - conventional therapies are high cost, insufficient pharmacokinetic data and less bioavailability.

There is existence of many signaling molecules yet to be investigated fully. Therefore, understanding of the signaling network of bacteria is essential for finding newer inhibitory factors for such communication. Future researchers for prevention of drug resistance can utilize this knowledge without exerting selective pressure on bacteria. This can also inhibit biofilm formation by microorganisms.

\section{Acknowledgement}

The author(s) gratefully acknowledge valuable support of Pravara institute of medical sciences (Deemed University) for conducting this study.

\section{References}

Arthur, M., Molinas, C., Courvalin, P. 1992. The van $S$ - van $R$ two component regulatory system controls synthesis of depsipeptide peptidoglycan precursors in Enterococcus facium BM4147. J Bacteriol. 174: 2582 2591.
Bokhove, M., Nadal, J. P., Quax, W. J., Dijkstra, B.W. 2010. The quorum quenching $\mathrm{N}$ aceyl - homoserine lactone acylase PvdQ is an Ntn hydrolase with an unusual substrate binding pocket. Proc Natt Acad Sci. U.S.A 107: 686 - 669.

Benghezal, M., Adam, E., Lucas, A., Burn, C., Michael, G.O., Christine, D. et al. 2007. Inhibitors of bacterial virulence identified in a surrogate host model. Cell Microbiol. 9: 1336 - 1342.

Bose, S., Ghosh, A. K. 2011. Biofilms: A challenge to medical science. J Clin Diag Res. 5(1): 127 - 130.

Bose, S., Ghosh, A. K. 2015. Diagnosis of biofilm associated infection in medical devices. Chapter 4. In: Biomedical and medical device associated infection. L. Barnes and I. R. Cooper, Editors. Wood house publication: Imprint of Elsevier. 71 79.

Chan, K. G., Steve, A., Kalai, M. Heg., Choon - Kook, S., Chhabra, S. R., Camara, M., et al. 2011. Characterization of $\mathrm{N}-$ acyl homoserine lactone degrading bacteria associated with zingiber officinale (ginger) rhizosphere: coexistence of quorum sensing and quorum quenching in Acinetobacter and Burkholderia. BMC. Microbiol. 11(1): 51.

Cugini, C., Calfee, M.W., Farrow, J.M., Morales, D.K., Pesci, E.C., Hogan, D.A. 2007. Farnesol, a common sesquiterpene, inhibits PQS production in Pseudomonas aeruginosa. Mol Microbiol. 65: 896 906.

David, L. P., Ann, M. S. 2005. Bacterial quorum sensing and bioluminescence. Asso Biology Lab edu. 27: 201 - 215.

Dong, Y. H., Xu, J. L., Li, X. Z., Zhang, L. H. 2000. AIIA. An enzyme that 
inactivates the acylhomoserine lactone quorum sensing signal and attenuates the virulence of Erwinia carotovora. Prac Natt Acad Sci. U.S.A. 97: $3526-$ 3531.

Dekimpe, V., Deziel, E. 2009. Revisiting the quorum sensing hierarchy in Pseudomonas aeruginosa: the transcriptional regulator $r h l r$ regulates lasr - specific factors. Microbiol. 155: $712-723$

Evers, S., Courvalin, P. 1996. Regulation of van B type vancomycin resistance gene expression by the van $S(B)-$ van $R C B$, two component regulatory system in Enterococcus faecalis v583, J Bacteriol. 178: 1302 - 1309.

Gilson, L., Kuo, A., Dunlap, P.V. 1995. AinS and a new family of autoinducer synthesis proteins. J. Bacteriol. 177:6946-6951

Ivanova, K., Fernandes, M.M., Tzanov, T. 2013. Current advances on bacterial pathogenesis inhibition and treatment strategies. @ Formatex. 322 - 336.

Jimenez, P. N., Koch, G., Thompson, J.A., Xavier, K.B., Robert, H.C., Quax, W. J. 2012. The multiple signaling systems regulating virulence in Pseudomonas aeruginosa. 2012. Microbiol Mol Biol Rev. 76(1): 46 65.

Kim, M.H., Choi, W. C., Kang, H. O., Lee, J.S., Kang, B.S., Kim, K.J. et al. 2005. The molecular structure and catalytic mechanism of a quorum - quenching $\mathrm{N}$ - acyl - L - homoserine lactone hydrolase. Proc Natt Acad Sci. U.S.A. 102(49): 17606 - 17611.

Kaufmann, G.F., Park, J., Mee, J.M., Ulevitch, R.J., Janda, K.D. 2008. The quorum-quenching antibody RS2 $1 \mathrm{G} 9$ protects macrophages from the cytotoxic effects of Pseudomonas aeruginosa quorum sensing signaling molecule $\mathrm{N}-3$ - Oxo - dodecanoyl homoserine lactone. Mol Immunol. 45: $2710-2714$.

Kaufmann, G.F., Sartorio, R., Lee, S.H., Mee, J.M., Attobell, L.J., Kiyawa, D.P. et al. 2006. N- acyl homoserine lactone - mediated bacterial quorum sensing. J Am Chem Soc. 128: 2802 2803.

Kiran, S., 2011. Enzymatic quorum quenching increases antibiotic susceptibility of multidrug resistant Pseudomonas aeruginosa. Irani $\mathbf{J}$ Microbiol. 3: 1 - 12.

Lerat, E., Moran, M.A. 2004. Evolutionary history of quorum sensing system in bacteria. Mol Biology Evolution. 21(5): $903-913$.

Lewis, H.A., Furlong, E. B., Laubert, B., Eroshkina, G.A., Batiyenko, Y., Adam, J.M. et al. 2001. A structural genomic approach to the study of quorum sensing: crystal structures of three LuxS orthologs structure. 9(6): $527-537$.

La Sarre, B., Federle, M.J. 2013. Exploiting quorum sensing to confuse bacterial pathogens. Microbiol Mol Biol Rev. 77(1): $73-111$.

Labbale, M., Queck, S.Y., Koh, K. S., Rice, S. A., Givskov, M., Kjelleberg, S. 2004. Quorum sensing controlled biofilm development in Serratia liquifaciens MGI. J Bacteriol. 186(3): $692-698$.

Lead better, J.R., Greenberg, E.P. 2000. Metabolism of aryl homoserine lactone quorum sensing signals by variovorax paradoxus. J Bacteriol. 182: 6921 - 6926.

Miller, M. B., Bassler, B. L. 2001. Quorum sensing in bacteria. Ann Rev. Microbiol. 55(1): 165 - 199.

Mayville, P., Guangyon, G., Beavis, R., Yang, H., Goger, M., Richard, P.N. et al. 1999. Structure - activity analysis of synthetic auto inducing thiolactone 
peptides from Staphylococcus aureus responsible for virulence. Proc Natl Acad Sci. U.S.A. 96: 1218 - 1223.

Maria, S.C., Wolfgang, D.B., Mengsheng, G., Jayne, B.R., Downie, J.A. 2007. Quorum sensing regulation in rhizobia and its role in symbiotic interaction with legumes. Philos Trans R Soc Lond B Biol Sci. 362(1483): 1149 1163.

Miyairi, S., Tateda, K., Fuse, E.T., Ueda, C. 2006. Immunization with 3-oxo dodecanoyl - 1 - homoserine lactone - protein conjugate protects mice for lethal Pseudomonas aeruginosa lung infection. J Med Microbiol. 55: 1381 $-1387$.

Miller, M. B., Skorupski, K., Lenz, D.H., Taylor, R. K., Bassler, B. L. 2002. Parallel quorum sensing systems converge to regulate virulence in Vibrio cholerae. Cell. 110: 303 - 314.

Nealson, K. H., Hastings, J. W. 1979. Bacterial bioluminescence: its control and ecological significance. Microbiol Rev. 43(4): 496.

Otto, M. 2004. Quorum sensing control in Staphylococci - a target for antimicrobial drug therapy? FEMS Microbiol Lett. 241: 135 - 141.

Parsek, M. R., Val, D.L., Hanzelka, B.L., Cronan, J.E., Greenberg, E.P. 1999. Acyl homoserine lactone quorum sensing signal generation. Proc Natl Aca Sci. U.S.A. 96: 4360 - 4365.

Rutherford, S.T., Bassler, B.L. 2012.
Bacterial quorum sensing: its role in virulence and possibilities for its control. Cold Spring Harb Perspect Med. 2(11): $1-25$.

Rasko, D. A., Sperandio, V. 2010. Anti virulence strategies to combat bacteria - mediated disease. Nat Rev Drug Discov. 9:117 - 128.

Saye, D.E. 2007. Recurring and antimicrobial - resistant infections: considering the potential role of biofilm in clinical practice. Ostomy Wound Manage. 53: 46 - 62.

Song, B.T., Wen, S. Y. 2013. Development of quorum - based anti - virulence therapeutics targeting Gram negative bacterial pathogens. Int $\mathrm{J}$ Mol Sci. 14: 16570 - 16599.

Uroz, S., Chhabra, S.R., Camara, M., William, P., Oger, P., Dessaux, Y. 2005. N-Acylhomoserine lactone quorum-sensing molecules are modified and degraded by Rhodococcus erythropolis W2 by both amidolytic and novel oxidoreductase activities. Microbiology. 151: 33133322.

Wynendaele, E., Bronselaer, A., Nielandt, J., D'Hondt, M., Stalmans, S., Bracke, N. et al. 2012. Quorumpeps database: chemical space, microbial origin and functionality of quorum sensing peptides. Nucleic acid research. pp 15 .

\section{How to cite this article:}

Seema Bose and Atindra Krishna Ghosh. 2016. Understanding of Quorum - Sensing: A Possible Solution for Drug Resistance in Bacteria. Int.J.Curr.Microbiol.App.Sci.5(2): 540-546. doi: http://dx.doi.org/10.20546/ijcmas.2016.502.061 